surprising as $C$ trachomatis is now the most common sexually transmitted infection in Britain. Other community based studies on inflammatory results of cytology have not found such a high prevalence, ${ }^{2}$ but comments made at the time suggested that the infection rates were lower than would be expected. ${ }^{3}$ The prevalence of infections was higher than reported in studies of patients with symptoms attending general practice and family planning clinics, where $C$ trachomatis was isolated in $8 \%$ and $9 \%$ respectively. ${ }^{+5}$ Indeed, the prevalence of $C$ trachomatis in our study group was similar to the $16 \%$ found in a study of screening before termination of pregnancy. ${ }^{6}$ Many doctors think that screening for sexually transmitted diseases should be performed before termination of pregnancy because of the high risk of the presence of a serious infection. We think that this would also be a sensible policy in patients who have had inflammatory changes on a smear as the recognition of asymptomatic infections such as $C$ trachomatis is very important. A three to six months' delay in the diagnosis of cervical intraepithelial neoplasia is probably of no long term detriment to the patient, but such a delay in the diagnosis and treatment of $C$ trachomatis cervicitis may result in long term complications of the upper genital tract and potential transmission of the infection to sexual partners.

There was a strong correlation between the presence of a sexually acquired infection and abnormalities on colposcopy. $C$ trachomatis was the only individual infection to show strong correlation. The prevalence of the other infections was much lower so that many more patients would need to be studied before such correlations would become evident for these infections.

The abnormality rates of $13 \%$ for cervical intraepithelial neoplasia and $25 \%$ for wart virus infection are comparable with those found in other studies on inflammatory cytology. ${ }^{12}$ Interestingly, we found that patients with abnormal results on colposcopy were significantly younger than those with normal findings. This is contrary to the belief that inflammatory changes are more likely to be found in older women than in younger women, and thus to have more serious implications. It is not surprising, though, that contraceptive use at the time of examination or partner change over the past year showed no significant correlation with abnormality on colposcopy. Presumably any sexually transmitted agent implicated in the aetiology of cervical intraepithelial neoplasia infects the cervix some time before producing premalignant changes.

As from April 1989, and since this study was initiated, the cytology reporting form has changed to form HMR 101/5 (1989). The working party of the British Society for Clinical Cytology recommended that simple inflammatory changes need not be reported, ${ }^{7}$ but severe inflammatory changes may now be reported to box 21 (cytology report) or as borderline changes; the management suggested is either to repeat the smear in so many months or to repeat after treatment when trichomonas, candida, or gardnerella have been identified. It is important to remember that $C$ trachomatis has no specific cytological features other than inflammatory changes ${ }^{x}$ and may coexist with these infections.

It is obviously impractical to suggest that all premenopausal women with one smear showing inflammatory changes be referred to a department of genitourinary medicine or a colposcopy clinic. These already overstretched clinics would be unable to cope with the increase in patients. We suggest that women with one such smear who are under 25 or have recently changed sexual partner should be referred to a department of genitourinary medicine because of the risk of sexually transmitted infection being present.

Most doctors now follow the recommendations of the British Society for Clinical Cytology working party and refer women with inflammatory changes on two consecutive smears to a colposcopy clinic. ${ }^{9}$ Most departments of genitourinary medicine now perform colposcopic examinations, and perhaps such patients should be referred there for both colposcopy and infection screening. Alternatively, when patients are seen at a colposcopy clinic we suggest taking, as a minimum, endocervical swabs for gonorrhoea and chlamydia in addition to colposcopy to exclude other readily treatable causes of inflammatory changes on a smear.

We thank Mrs C Gibson for typing the manuscript and Peter Williams for his help with statistical analysis.

1 Toon PG, Arrand JR, Wilson LP, Sharp DS. Human papillomavirus infection of the uterine cervix of women without cytological signs of neoplasia. $\mathrm{BrMed}$ 1986;293:1261-4

2 Kirkman RJE, Peel JM, Fenton DW, Sharp F. A pilot study of the benefit of colposcopy as a further screening procedure within the community health service. Community Med 1986;8:240-4.

3 Walkinshaw SA, Roberts ADG, Cordiner JW. Human papillomavirus infection of the uterine cervix of women without cytological signs of neoplasia BrMed 1 1987.294:117.8.

4 Southgate LJ, Treharne JD, Forsey T. Chlamydia trachomatis and Neisseria gonorrhoea infections in women attending inner city general practices. gonorrhoea infections in wor

5 Handsfield $\mathrm{HH}$ Jasman LL, Roberts PL, et al Criteria for selective screening for Chlamydia trachomatis infection in women attending family planning clinics. 7A.MA 1986;255:1730-

6 Hill LVH, Luther ER, Young D, Pereira L, Embil JA. Prevalence of lower genital tract infections in pregnancy. Sex Transm Dis 1988;15:5-10.

7 Evans DMD, Hudson EA, Brown CL, et al. Terminology in gynaecological cytopathology: report of the working party of the British Society for Clinical Cytology. F Clin Pathol 1986;39:933-44.

8 Geerling S, Nettum JA, Lindner LE, et al. Sensitivity and specificity of the Papanicolaou stained cervical smear in the diagnosis of chlamydial trach matis infection. Acta Cvtologica 1985;5:671-5.

9 BSCC Working Party on Terminology in Gynaecological Cytopathology. Management of women with abnormal cervical smears. Bulletin of the Royol College of Pathologists. 1986; No 56:1-2.

(Accepled 3 fanuary 1990)

\title{
Intravenous use of slow release morphine sulphate tablets
}

Substance Abuse Unit, City General Hospital, Stoke on Trent, Staffordshire ST 4 6QG R N Bloor, MRCPSYCH, consultant N J F Smalldridge, MRCPSYCH, clinical assistant

Correspondence to: Dr Bloor.

$\operatorname{BrMed}$ f 1990;300:640-1

\section{R N Bloor, N J F Smalldridge}

Slow release morphine sulphate tablets are given to relieve severe pain and are prescribed for treatment at home. Misuse of this drug may have similar implications for prescribers to those reported for other drugs such as temazepam, buprenorphine, and cyclizine. Buprenorphine is reported to be the preferred drug of over half of intravenous drug users in contact with drug services in eastern Glasgow, and this figure rises to $69 \%$ when use of buprenorphine in combination with other drugs such as temazepam is included.' We could not find any reports on intravenous misuse of slow release morphine sulphate tablets or on the amount of morphine that can be extracted from them. We report three cases of such misuse by people who obtained the drug on prescription or "on the street," and the results of our reproduction of the extraction procedure.

\section{Case reports, method, and results}

Case 1-A 26 year old man with a six year history of dependence on opiates presented after his use of opiates had increased over three months. He had progressed from oral to intravenous use and was using slow release morphine sulphate tablets, which he 
crushed to produce a morphine sulphate extract. $\mathrm{He}$ had increased his use from low dose to $300-400 \mathrm{mg}$ daily.

Case 2-A 20 year old man presented to this unit with a seven month history of heroin misuse. He had injected heroin daily for four months and had subsequently inhaled it. For three weeks before presentation he had been injecting an extract of $100 \mathrm{mg}$ slow release morphine sulphate tablets daily.

Case 3-A woman aged 26 presented with a long history of intravenous use of heroin. After treatment she had been free of drugs for one year. Inoperable carcinoma of the cervix was then diagnosed, and she was given slow release morphine sulphate $(60 \mathrm{mg}$ three times a day) for abdominal pain. The tumour was successfully treated with radiotherapy, but her use of slow release morphine sulphate tablets increased to $240 \mathrm{mg}$ a day. She started extracting morphine from the tablets and injected it intravenously eight to 12 times a day.

Amount of morphine sulphate ( $\mathrm{mg}$ ) extracted from $60 \mathrm{mg}$ tablets of slow release morphine sulphate by five inexperienced volunteers

\begin{tabular}{lccccc}
\hline & $\begin{array}{c}\text { Subject } \\
1\end{array}$ & $\begin{array}{c}\text { Subject } \\
2\end{array}$ & $\begin{array}{c}\text { Subject } \\
3\end{array}$ & $\begin{array}{c}\text { Subject } \\
4\end{array}$ & $\begin{array}{c}\text { Subject } \\
5\end{array}$ \\
\hline First attempt & 36.52 & $45 \cdot 38$ & 49.32 & 37.82 & $38 \cdot 00$ \\
Second attempt & 22.42 & 44.30 & 45.56 & 36.08 & 33.80 \\
\hline
\end{tabular}

With the cooperation of someone who had used slow release morphine sulphate tablets we recorded the extraction procedure on videotape. The recording was used to guide three nursing staff and two medical students with no previous experience of the extraction method in preparing two extracts from $60 \mathrm{mg}$ tablets of slow release morphine sulphate. The subjects used procedures and equipment identical with those shown in the recording to reproduce "street" conditions as closely as possible. The extracts were analysed for morphine sulphate by high performance liquid chromatography.

The table shows the results of the extraction pro- cedure. The subjects produced an extract with a mean content of $38.92 \mathrm{mg}$ (range $22.42-49.32 \mathrm{mg}$ ) - that is, $65 \%$ of the available morphine sulphate. One sample was subjected to a second extraction (users often reprocess the residue from the original extraction) and this produced $15 \%$ increase in the amount of morphine sulphate.

\section{Comment}

Slow release morphine sulphate can be misused intravenously, and the three patients reported on had a high intake within a short time. Our results suggest that intravenous users manage to extract $65 \%$ of the available morphine sulphate and the dose of methadone prescribed for detoxification should be based on this figure.

Increasing misuse of prescribed drugs for intravenous use has been reported with temazepam, buprenorphine, and cyclizine. ${ }^{2-4}$ Sakol et al suggested that the formulation of a drug affects its potential for abuse and that prescribers have an obligation to consider this. ${ }^{5}$ Buprenorphine is now a controlled drug, and two manufacturers of temazepam have stopped making injectable preparations. We emphasise the need for caution when prescribing opiates of any form and the importance of checking whether a patient has a history of drug misuse before prescribing drugs that may be misused.

We thank Mr K Hale of the Regional Laboratory for Toxicology, Birmingham, for analysing the extracts of morphine sulphate.

1 Gray RF, Ferry A; Jauhar P. Emergence of buprenorphine dependence. $\mathrm{Br} \mathcal{J}$ Addict 1989;84:1373-4.

Stark CR Sykes R, Mullin PJ Temazepam abuse Lancet 1987:ii-802-3.

3 O'Connor JJ, Maloney E, Travers R, Campbell A. Buprenorphine abuse among Connor JJ, Maloney E, Travers R, Campbell

4 Ruben SM, McLean PC, Melville J. Cyclizine abuse among a group of opiate Ruben SM, McLean PC, Melville J. Cyclizine abuse among a g
dependants receiving methadone. Br $\mathcal{F}$ Addict 1989;84:929-34.

5 Sakol MS, Stark C, Sykes R. Buprenorphine and temazepam abuse by drug takers in Glasgow - an increase. $\mathrm{Br} \mathcal{F}$ Addict 1989;84:439-41.

(Accepted 29 November 1989)

\section{Spontaneous remission of hepatocellular carcinoma after massive gastrointestinal haemorrhage}

\section{G Tocci, A Conte, P Guarascio, G Visco}

Lazzaro Spallanzani Hospital for Infectious Diseases, 00149 Rome, Italy

$\mathrm{G}$ Tocci, MD, registrar

A Conte, MD, registrar

P Guarascio, MD, senior registrar

G Visco, MD, consultant

Correspondence to: Dr Tocci.

BrMed f 1990;300:641-2
Spontaneous remission of hepatocellular carcinoma is extremely rare. ${ }^{1}$ We describe spontaneous remission of hepatocellular carcinoma after a massive intercurrent gastrointestinal haemorrhage.

\section{Case report}

A 79 year old man had been attending our outpatient clinic since January 1980 with active liver cirrhosis secondary to non-A, non-B hepatitis. He received immunosuppressive treatment with prednisone $(7.5 \mathrm{mg}$ daily) and azathioprine (50 mg daily) from January to March 1980; the treatment was then altered to azathioprine alone (100 mg daily) because of gastric discomfort. All treatment was stopped in March 1983 after a second liver biopsy showed an increase in the histological signs of activity.

In June 1984 routine periodic radioimmunoassay showed an increased serum concentration of $\alpha$ fetoprotein $(100 \mu \mathrm{g} / 1)$. Ultrasonography and computed tomography of the liver showed a round dense image, which was about $5 \mathrm{~cm}$ in diameter and had a necrotic core near the hepatic hilum. A liver biopsy guided by ultrasonography disclosed a microtrabecular hepatocarcinoma. His general condition deteriorated during the following months; he developed jaundice, and the serum concentration of $\alpha$ fetoprotein increased to $625 \mu \mathrm{g} / \mathrm{l}$. No treatment was attempted because of his explicit, informed refusal.

In September 1984 his general condition deteriorated further and he was admitted to another hospital, where treatment with high doses of intravenous hydrocortisone ( $2 \mathrm{~g}$ daily) was begun. A few days later he had a massive haematemesis followed by severe and prolonged shock. His haemoglobin concentration fell below $40 \mathrm{~g} / \mathrm{l}$ for several hours, and endoscopy disclosed a bleeding gastric ulcer. He recovered from shock after the transfusion of 3 litres of fresh blood. His condition subsequently improved and his jaundice receded. Five months later ultrasonography of the liver showed a surprising reduction in the size of the tumour. In addition, his serum concentration of $\alpha$ fetoprotein had fallen to $165 \mu \mathrm{g} / \mathrm{l}$.

Ultrasonography and computed tomography in May 1985 showed a further reduction in the size of the tumour, and his serum $\alpha$ fetoprotein concentration was normal $(5 \mu \mathrm{g} / \mathrm{l})$. The tumour had completely disappeared by early 1987 , and this was confirmed by computed tomography in 1988. Microcalcifications were observed at the former site of the tumour. 